

HTTP://DX.DOI.ORG/10.22256/PUBVET.V11N2.154-158

\title{
Teniase e Cisticercose
}

\section{Daniela Ferreira \& Fernanda Lúcia Alves Ferreira}

\section{Universidade Anhanguera de São Paulo - Campus Abc, São Bernardo do Campo, Brasil. \\ *Autor para Correspondência, E-mail: veterinariadaniela@live.com}

\begin{abstract}
RESUMO. $O$ foco deste trabalho é mostrar os cuidados que a população deve ter para que não se contamine com teníase e cisticercose. Alertando-a, deste modo, sobre o ciclo da contaminação e, por conseguinte, o desenvolvimento da doença. Com este objetivo, revisaremos o ciclo evolutivo da epidemiologia, enfatizando a patogenia com suas sintomatologias e tratamento. Considerada uma zoonose de grande impacto à saúde pública e econômica, pois, devido à forma descuidada com que as vísceras e carcaças do abate animal são descartadas, promovem a contaminação de rios, lagos e fontes de abastecimento público de água e, deste modo, contaminando, sobretudo, as populações de regiões nas quais o tratamento sanitário é precário ou inexistente. Tal contaminação se dá através de ovos de cisticerco eliminado nas fezes humanas e animal, provenientes da Taenia solium e Taenia saginata. Frisamos a divulgação e conscientização pública, principalmente nas regiões mais carentes de inspeção sanitária dos alimentos e abatedouros.
\end{abstract}

Palavras-chave: Zoonese, taenia solium, taenia saginata, cisticerco, inspeção sanitária

\section{Taeniasis and cysticercosis}

ABSTRACT. The focus of this work is to show the care that people should not to be polluted with taeniasis and cysticercosis. Alerting the thus contamination of the cycle and hence the development of the disease. To this end, we will review the life cycle of the epidemiology, pathogenesis emphasizing with their symptomatology and treatment. Considered a zoonosis of great impact to the public and economic health because, due to the careless way in which the viscera and animals slaughtered carcasses are discarded, promote the contamination of rivers, lakes and sources of public water supply and thus defiling above all, the population of regions in which the health treatment is poor or nonexistent. Such contamination occurs through cysticercosis eggs eliminated in the human or animal feces, from Taenia solium and Taenia saginata. We stress the dissemination and public awareness, particularly in the poorest regions of sanitary inspection of food and slaughterhouses.

Key-words: Zoonosis, taenia solium, taenia saginata, cysticercosis, sanitary inspection

\section{Teniasis y cisticercosis}

RESUMEN. El objetivo de este trabajo es mostrar el cuidado que las personas deben tener para no ser contaminadas con la teniasis y la cisticercosis. Alertándolas, de este modo, sobre el ciclo de la contaminación y, por lo tanto, el desarrollo de la enfermedad. Con este fin, revisaremos el ciclo evolutivo de la epidemiología, enfatizando la patogénesis con su sintomatología y tratamiento. Considerada como una zoonosis de gran impacto para la salud pública y económica, ya que, debido a la forma descuidada en el que las vísceras y las canales de animales sacrificados se descartan, promueven la contaminación de los ríos, lagos y fuentes de suministro de agua pública y, de esta forma, contaminando, sobre todo, la población de regiones en las que el tratamiento sanitario es precario o inexistente. Tal 
contaminación se produce a través de los huevos de cisticercos eliminados en las heces humanas o de animales, provenientes de la Taenia solium y Taenia saginata. Hacemos hincapié en la divulgación y concientización del público, principalmente en las regiones más carentes de inspección sanitaria de los alimentos y los mataderos.

Palabras clave: Zoonosis, Tenia solium saginata, cisticercosis, la inspección sanitaria

\section{Introdução}

Endoparasitas intestinais é um problema que afeta tanto a medicina veterinária com medicina humana, sendo hospedeiro intermediário nos suínos e bovinos infectados, no caso da Taenia soluim e Taenia saginata, e ao homem sendo hospedeiro definitivo. (Pfuetzenreiter and Pires, 2000, Acevedo Nieto, 2015).

O Brasil é um dos países que apresenta maior número de parasitoses humanas em relação a outros países do mundo, devido ao seu caráter de país em desenvolvimento e aos seus aspectos políticos e sócios econômicos. Neste contexto, em especial em estados com maiores índices de pobreza e de distribuição de renda irregular, como os estados das regiões Norte e Nordeste, muitas parasitoses tornam-se ainda mais graves. As helmintoses são o tipo de parasitose mais frequente em todo o mundo e, nestas regiões do país, tornam-se um grande problema de saúde pública. (Soares et al., 2015, Santos and Barros, 2009)

Tratando-se de uma zoonose que eventualmente atinge o homem, o complexo teníase/cisticercose preocupa vários profissionais da área de saúde, como os infectologistas, neurologistas, oftalmologistas, sanitaristas e médicos veterinários. Contudo, a melhor forma de prevenção é o esclarecimento divulgação desta zoonose e medidas de inspeção sanitária, juntamente com saneamento básico, que é de total e indispensável direito da população (Souza et al., $\underline{2013}$ ).

\section{Morfologia}

Classe Cestoda, de corpo achatado sem canal digestivo, com corpo segmentado, cada segmento contendo um ou dois conjuntos de órgãos reprodutores, masculinos e femininos (Urquhart, 1996).

\section{Ordem Cyclosphyllidea}

Sua estrutura e função são constituídas de cabeça ou escoléx com órgãos de fixação, pescoço curto não segmentado e uma cadeia de segmentos, conhecida como estróbilo e cada segmento como proglote, e os órgãos de fixação são quatro ventosas do lado do escólex e podendo conter ganchos, o escólex geralmente apresenta um rostelo em algumas espécies que podem também conter uma ou mais fileiras de ganchos para fixação, já os proglotes desenvolvem-se na região do colo e se tornam sexualmente maduros na porção final do estróbilo e cada proglote é hermafrodita, e com um ou dois conjuntos de órgãos reprodutores, e os poros genitais abrem-se nas bordas laterais do segmento, assim como mostrado na (Figura 1a); tanto pode ocorrer autofecundação, ou fertilização cruzada entre proglotes, à medida que o segmento esta amadurecido, sua estrutura interna desaparece amplamente e o proglote maduro ou grávido irá conter apenas resquícios do útero, ramificado e cheio de ovos, e geralmente os segmentos grávidos saem intactos dos estróbilos sendo eliminados pelas fezes, e já fora do corpo, os ovos são liberados por desintegração do segmento. Veja a (Figura 2b) (Urquhart, 1996).

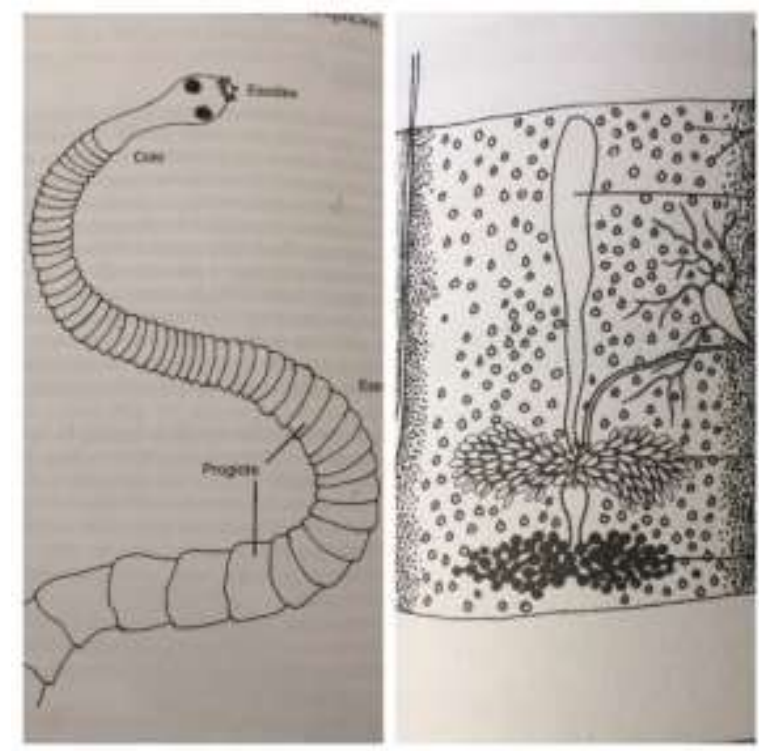

Figura 1. Estrutura de um cestoide ciclofilídeo típico e escólex, colo e estróbilos. Fonte: (Urquhart (1996).

Sendo o ovo constituído de embrião hexacanto ou oncosfera e uma casca espessa e escura, levemente estriada denominada embrióforo. Já o tegumento do cestoide adulto é altamente absorvente, assim o verme adquire toda nutrição pela estrutura e o sistema excretor é composto de 
células ativas que levam a canais eferentes que seguem pelos estróbilos, assim descarregando no segmento terminal. E sua forma larval como Taenia solium é Cysticercus cellulosae, e como Taenia saginata a forma larval é Cysticercus bovis (Urquhart, 1996).

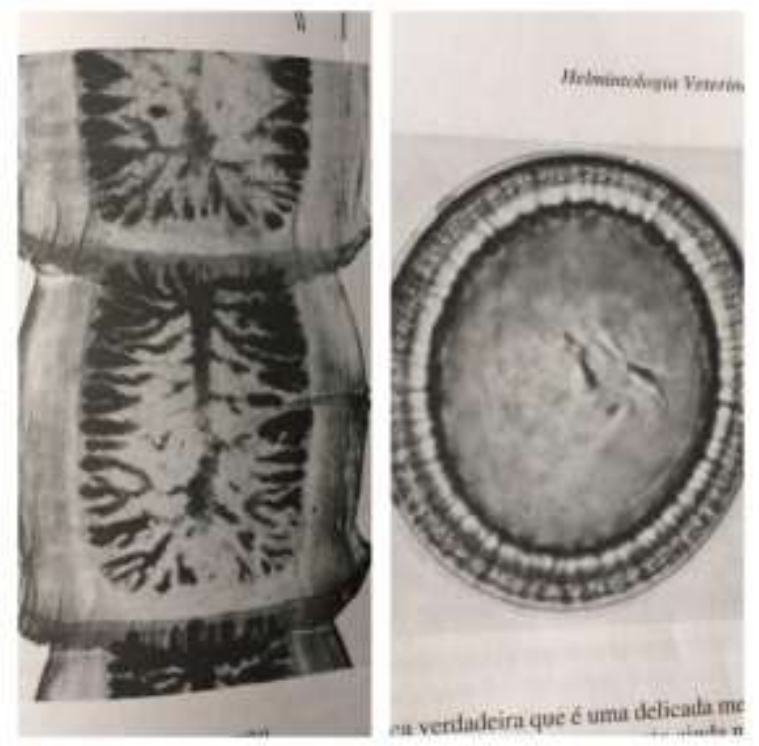

Fígura 2. Segmento maduro mostrando órgãos reprodutores segmentado grávido e ovo de tenídeo típico. Fonte: Urquhart (1996).

\section{Dimensão}

Taenia solium mede de 2 a $3 \mathrm{~m}$ de comprimento, podendo, entretanto, atingir até $8 \mathrm{~m}$ de comprimento por 5 a $8 \mathrm{~mm}$ de largura. Hospedeiro definitivo é o homem e os intermediários suínos e raramente, caninos, felinos, ruminantes equinos e homem.

Taenia saginata mede de 3 a $8 \mathrm{~m}$ de comprimento, podendo atingir até $12 \mathrm{~m}$ por 5 a 7 $\mathrm{mm}$ de largura. Sendo hospedeiro definitivo o homem e hospedeiros intermediários, bovinos, raramente ovinos e caprinos e excepcionalmente o homem (Fortes, 2004).

\section{Etiologia}

Taenia soluim o hospedeiro definitivo é o homem, se infectando pela ingestão de carne de porco malcozida ou malpassada, cotendo cisticercos (Cysticercus cellulosae) vivos, e o suíno hospedeiro intermediário, se infectando ao ingerir água contaminada ou com ovos embrionados de Taenia solium, mas também o homem pode contrair a cisticercose ao ingerir ovo de $T$. solium, levando diretamente a boca através das mãos sujas ou verduras e frutas contaminadas, cru ou mal lavadas e nos casos de autoinfecção por antiperistaltismo (Narata et al., 1998). Já a Taenia saginata o homem o hospedeiro definitivo e se infecta com ingestão de cisticercos contidos em carne bovina crua ou malpassada, e o bovino é o hospedeiro intermediário, se infectando ao pastar em campos contaminados com ovos de T. saginata ou ao beber água poluída com embrióforos da tênia mencionada, porém o homem pode contrair a cisticercose pela ingestão de ovos de $T$. saginata contidos em verduras e frutas consumidas crus e mal lavadas ou levadas diretamente a boca como no caso da T. solium (Fortes, 2004).

\section{Ciclo evolutivo}

Taenia solium o ciclo evolutivo é indireto com o hospedeiro intermediário, salvo exceções o cestoide adulto é encontrado no intestino delgado, do hospedeiro definitivo, e os segmentos e ovos atingem as fezes, usando ganchos, a mucosa é lacerada atingindo assim a corrente sanguínea ou linfática, e uma vez no seu local de predileção a oncosfera perde os ganchos e se desenvolve (Veiga et al., 2006, Lyra and Silvia, 2002).

Taenia saginata o homem infectado pode eliminar milhões de ovos ao dia pelas fezes ou como segmentos intactos, com cada um contendo 25.000 ovos, que podem sobreviver na pastagem durante meses, e após a ingestão por bovino a oncosfera segue através do sangue para a musculatura estriada, podendo ser visível por cerca de 2 semanas, em forma de ponto pálido semitransparente de aproximadamente $1 \mathrm{~mm}$ de diâmetro, não sendo infectante para o homem até 12 semanas, após atingir seu tamanho Maximo de $1 \mathrm{~cm}$, Já é envolto por uma cápsula fibrosa fina, e sua longevidade varia de semana a anos, quando morre é substituída por uma massa caseosa friável que pode tornar-se calcificada. E tanto os cistos vivos e mortos estão presentes na mesma carcaça e se o homem ingerir a carne crua ou malpassada será infectado (Monteiro, 2011).

\section{Patologias e sintomalogias clinicas}

Taenia solium com sua ação no homem, seu hospedeiro definitivo é mínima, com espoliação pouco significativa, assim como a ação traumática devido aos acúleos e ventosas, porém o movimento do rostelo e acúleos pode provocar irritação das terminações nervosas do plexo simpático, consequentemente podendo surgir reflexos, tais como alergias, convulsões e até ataque epileptiforme. Porém depois de um determinado tempo, (em media três meses) os cisticercos sofrem degenerescência calcária. No 
caso da Taenia saginata os sintomas são semelhantes (Arruda et al., 2006, Bürger et al., 2015).

\section{Epidemiologia}

Acontece em dois modelos bem distintos como, cidades e países em desenvolvimento e desenvolvidos, tanto com a Taenia solium ou Taenia saginata podemos coloca-las com este padrão, referente ao nível social e condições de instalações sanitárias, vem o grau de informação e contaminação de acordo com as condições econômicas, criadores de fundo de quintal, costumes rurais como defecar no fundo do quintal, inspeção em matadouros, abatedouros clandestinos, ingestão de carnes cruas ou malpassadas e eliminação de carcaças (Corrêa et al., 1997, Takayanagui and Leite, 2001).

\section{Diagnóstico}

Taenia solium e Taenia saginata, ambas com o mesmo tratamento clinico, laboratorial e inspeção.

\section{Tratamento clínico}

Normalmente o paciente já faz o diagnostico ao verificar a presença de proglotes que são expulsas nas fezes.

\section{Laboratorial}

Ao suspeitar de teníase, é preciso pesquisar proglotes nas fezes, sendo assim feita a realização do exame laboratorial.

\section{Inspeção}

Constatado no animal morto a presença de cisticercos, principalmente no coração, músculos mastigadores, língua e outros órgãos, no caso da $T$. solium, diferenciando apenas que a $T$. saginata não se deve ingerir carne bovina crua, malpassada ou malcozida (Gomes et al., 2008).

\section{Tratamento}

$\mathrm{O}$ uso do fármaco Albendazol $^{\circledR}$ e Mebendazol® tem sido administrado nos casos da Taenia solium, já a Taenia saginata apenas o Albendazol®. Demonstrando eficácia em ambos os casos (Gomes et al., 2008)

\section{Controle}

Taenia saginata a higiene humana, cozimento dos alimentos a um ponto térmico maior que $57^{\circ}$ C, inspeção da carne e praticas gerais de higienização são eficazes, contamos também com o congelamento das carcaças infectadas a $-10^{\circ} \mathrm{C}$ durante 10 dias, sendo suficiente para destruir os cisticercos, porém este processo implica no valor econômico da carne e para $T$. solium é o mesmo precedimento, porém disso a exclusão de suínos em contato com fezes humanas (Corrêa et al., 1997).

\section{Considerações finais}

Em virtude ao que foi mencionada, devido às medidas de controle de inspeção e qualidade, nos matadouros, frigoríficos, carnes malpassada, malcozidas, má higienização de frutas e verduras, costumes rurais de moradores que defecam no quintal, levando assim a contaminação são de extrema importância, como o descarte ideal de carcaças, não as jogando em rios, córregos, lagos, para que não venham a contaminar a água que a população mais carente usa para próprio consumo. Seguido de informações para o tratamento para os humanos já afetados pela infecção, para ter melhor qualidade de vida ou cura.

\section{Referências Bibliográficas}

Acevedo Nieto, C. E. 2015. Complexo teníasecisticercose em assentamentos da Reforma Agrária do estado de Minas Gerais, Brasil. Departamento de Veterinária. Universidade Federal de Viçosa, Viçosa.

Arruda, P. S. P., Monteiro, L. L., Dias, F. S. \& Pinto, M. S. 2006. Cisticercose suina: aspectos clinico-epidemiologicos, imunodiagnostico e controle. Bioscience Journal, 20, 93-103.

Bürger, K. P., Buzza, P. L. T., Neto, E. B., dos Santos, L. R., Rossi, G. A. M. \& Martins, A. M. C. V. 2015. Complexo teniose-cisticercose: ocorrência em abatedouro de bovinos e conhecimento de estudantes do ensino médio e consumidores no Estado de São Paulo, Brasil. Revista Brasileira de Ciência Veterinária, 22, $1-10$.

Corrêa, G. L. B., Adams, N. A., Angnes, F. A. \& Grigoletto, D. S. 1997. Prevalência de cisticercose em bovinos abatidos em Santo Antônio das Missões, RS, Brasil. Revista da Faculdade de Zootecnia e Veterinária, 4, 4345.

Fortes, E. 2004. Parasitologia veterinária, 4 edn. Editora ícone, São Paulo.

Gomes, A. P., Nunes, E. R., Felippe, K. C., Carneiro, M. C. \& Santos, S. S. 2008. Teníase e cisticercose: breve revisão dos aspectos gerais. Pediatria, 44, 151-156. 
Lyra, T. M. d. P. \& Silvia, J. A. d. 2002. O componente social e sua importância na planificação em saúde animal. Revista CFMV, $8,11-20$.

Monteiro, S. G. 2011. Parasitologia na medicina veterinária. Roca, São Paulo.

Narata, A., Arruda, W., Uemura, E., Yukita, S., Blume, A. G., Suguiura, C. \& Pedrozo, A. 1998. Diagnóstico tomográfico em pacientes neurológicos. Arquivo Neuropsiquiatrico, 56, 245-249.

Pfuetzenreiter, M. R. \& Pires, F. D. Á. 2000. Epidemiology of teniasis/cysticercosis by Taenia solium and Taenia saginata. Ciência Rural, 30, 541-548.

Santos, J. M. G. \& Barros, M. C. R. B. 2009. Cysticercus bovis e Cysticercus cellulosae: Endoparasitas de importância no comércio da carne. Revista em Agronegócio e Meio Ambiente, 2, 21-39.

Soares, L. M., Oliveira, D. H. B., Ribeiro, A. A. M., Almeida, V. P. A., Freitas, D. R. J. \& Melo Evangelista, L. S. 2015. Complexo TeníaseCisticercose na visão de alunos da graduação. Revista Prevenção de Infecção e Saúde, 1, 1117.

Souza, L., Almeida, L. P. \& Carrijo, F. K. 2013. O complexo teníase-cisticercose: indicadores de fatores de risco em propriedades rurais com a criação artesanal de suínos em UberlândiaMG. Veterinária Notícias, 19, 1-10.

Takayanagui, O. M. \& Leite, J. P. 2001. Neurocysticercosis. Revista da Sociedade Brasileira de Medicina Tropical, 34, 283-290.

Urquhart, G. M. 1996. Parasitologia veterinária, 2 ed. Guanabara Koogan, Rio de Janeiro.

Veiga, C. F., Doro, D. L., Oliveira, K., M. P. \& Bombo, D. L. 2006. Estudo das condições sanitárias dos estabelecimentos comerciais de manipulação de alimentos do município de Maringá, PR. Revista Higiene Alimentar, 20, 28-35.

\section{Article History:}

Received 7 November 2016

Accepted 8 December 2016

Available on line 31 January 2017

License information: This is an open-access article distributed under the terms of the Creative Commons Attribution License 4.0, which permits unrestricted use, distribution, and reproduction in any medium, provided the original work is properly cited. 